\title{
SPATIAL-TEMPORAL CONDITIONS OF THE MOON BISTATIC SOUNDING
}

Olga V. Yushkova, Vyacheslav V. Yushkov, Roman A. Rudamenko, Taisiya N. Dymova

Kotelnikov Institute of Radioengineering and Electronics of RAS, Fryazino Branch, http:// fire.relarn.ru Fryazino 141190, Moscow Region, Russian Federation

o.v.y@mail.ru,iushkov@physics.msu.ru,crasher22@yandex.ru,tasinidze@gmail.com

Absract. In a framework of the Russian project "Luna-Resurs", the study of the lunar soil using bistatic radio sounding involving the Irkutsk Incoherent Scatter Radar is envisaged. Measurements are planned in the "up link" mode. According to the Fresnel approximation, the region of greatest reflection is defined as a region on the surface whose center is determined from the condition of equality of angles between the normal to the surface and directions from the base of the normal to the receiver and transmitter. The linear dimensions of the reflection region are associated with the Fresnel zones. Measurements are considered optimal if the region of greatest reflection falls within the visibility range of the orbital radar. The distance from the spacecraft to the center of the radio reflection region depends on the ballistic coordinates of the spacecraft, sometimes it equals to 500 kilometers or more, which must be taken into account when experiments are planned. The article presents the derivation of the formula, which determines the optimal time of reception of the reflected signal by the orbital radar for the bistatic location of the Moon in the "up link" mode. Keywords: bistatic radar, coordinates, reflected spot, the Moon, spacecraft

UDC 523.34-83

Bibliography - 6 references

Received 13.03.2019, accepted 29.03.2019

RENSIT, 2019, 11(1):13-20

DOI: $10.17725 /$ rensit.2019.11.013

CONTENTS

1. INTRODUCTION (13)

2. Technical parameters of Ground and ORBITAL RADARS (14)

3. SCHEME Of Bistatic REMOTE SENSING OF THE MOON IN "UP LINK" MODE (15)

4. LOCATION OF THE VISIBILITY ZONE OF THE ORBITAL RADAR AND THE REFLECTING SPOT OF RADIOSIGNAL (17)

5. Time of registrating of Reflected SIGNAL WITH ORBITAL RADAR (18)

6. Conclusion (18)

REFERENCES (19)

\section{INTRODUCTION}

The main aim of the Russian mission "Luna-Resource" is to study the structure and mineralogical composition of the upper cover of the Moon. Radiolocation is one of the remote methods that allow achieving the goal. To study the Moon surface with radiophysical methods, the radar complex RLK-L will be installed on board the Luna-26 orbiter, the launch of which is planned for 2023 [1]. The device operation is provided both in monostatic and in bistatic mode in conjunction with the Irkutsk Incoherent Scatter Radar (IRNR) of the Institute of Solar-Terrestrial Physics of the Siberian Branch of Russian Academy of Sciences[2].

Bistatic sounding of the Lunar soil were carried out from the spacecraft "Luna 10, 11, 12, 14, 19, 22", "Lunar orbiter 1", "Explorer 35", "Apollo 14,15,16", "Clementine", the radio signals were generated by the transmitters of the service radio complexes of satellites, and the echo was transmitted out on Earth. This location 
of receiving and transmitting equipment for Lunar probing was called "down link", unlike the "up link" scheme tested in 2006, when the radio complex of the Arecibo observatory was used as a transmitter, and radio signals were received on the "Lunar Reconnaissance Orbiter spacecraft (LRO)". Since the resolution of radar depends on the transmitter's energy potential, generation on Earth makes it possible to use a highpower radio signal for sensing, which is not limited to energy consumption norms and electromagnetic compatibility requirements with sensitive scientific and service devices. Thus, the peak power of the Arecibo chirp signal with a center frequency of $2379.2 \mathrm{MHz}$ with a deviation of $1.6 \mathrm{MHz}$ corresponded to $200 \mathrm{~kW}$. In the bistatic location of the moon, planned for under the Luna Resource mission, the IRNR will generate a chirp signal in the frequency range from 154 to $162 \mathrm{MHz}$ with a peak power of $3.2 \mathrm{MW}$, and its reflection from the Moon will be recorded by the RLK-L device.

There are techniques that allow determining the properties of the soil layer in the region of the reflection of radio waves based on a comparison of the characteristics of the emitted and received signals [3]. The main problem of the moon's bistatic radar experiments is the difficulty of defining the zone that forms the reflected radio signal, its distance from the point under the spacecraft and, as a result, in the absence of the possibility of parallel recording of the reflective surface of video and photo equipment. One of the particular problems of this problem is the choice of the optimal reception time by the orbital radar of the reflected signal: a signal too short in time may not contain useful information, and an unnecessarily long one requires allocating too much onboard processor memory and additional time resources when broadcasting scientific information to Earth. This article discusses issues related to determining the optimal time of reception of the reflected signal by the orbital radar, which depends on its location over the Moon, that is, on the coordinates of the spacecraft. Ballistic parameters of a spacecraft - its height $H_{s}$, longitude $\varphi_{s}$ and latitude $\theta$ are determined by the Flight Control Center and are provided for processing the measurement results in the selenographic coordinate system.

\section{TECHNICAL PARAMETERS OF GROUND AND ORBITAL RADARS}

Irkutsk incoherent scatter radar is under the jurisdiction of the Institute of SolarTerrestrial Physics of the SB RAS. It is a monostatic, pulsed radar with frequency scanning in the North-South direction in the sector $\pm 30^{\circ}$ by changing the carrier frequency in the range 154-162 MHz. The peak power of the generated radio signal reaches 3.2 MW, the repetition frequency of the probe pulses is $25 \mathrm{~Hz}$. The duration of the probe pulse is from 70 to $900 \mu \mathrm{s}$, the antenna gain is about $35 \mathrm{~dB}$.

The radio complex RLK-L, installed on the spacecraft "Luna-26", consists of two radar - radar-20 and radar-200. The device is designed to study the top layer of soil in the mode of monostatic location. Initially, the frequency range from 17.5 to 22.5 $\mathrm{MHz}$ was selected for the Radar-20, and from 175 to $225 \mathrm{MHz}$ for the Radar-200. Then it was decided to add the monostatic regime of the moon's radar with bistatic measurements. Since it is planned to use IRNR as a transmitter, to ensure joint 
operation with ground equipment, the frequency range of the Radar-200 is reduced to $140-180 \mathrm{MHz}$.

\section{SCHEME OF BISTATIC REMOTE SENSING OF THE MOON IN "UP LINK" MODE}

Bistatic measurements are carried out while the Moon and the spacecraft are in the scanning sector of the ground-based radar. Fig. 1 shows the location of the receiver and transmitter during the bistatic measurements of the Moon according to the "up link" mode: the number I indicate Earth, the number II indicates the Moon, and the III indicates the scanning sector of the ground-based radar. In the same figure, the number 1 indicates the path of the "direct" signal propagating from the ground-based radar to the spacecraft; and 2 - the path of the "reflected" signal, which passes the distance from the Earth to the Moon and, reflecting from the Moon, to the spacecraft. The center of the reflection region is found from the condition that the angles between the normal to the surface of the Moon and the direction to the Earth and between the same normal and the direction to the spacecraft are equal (Fresnel law). In

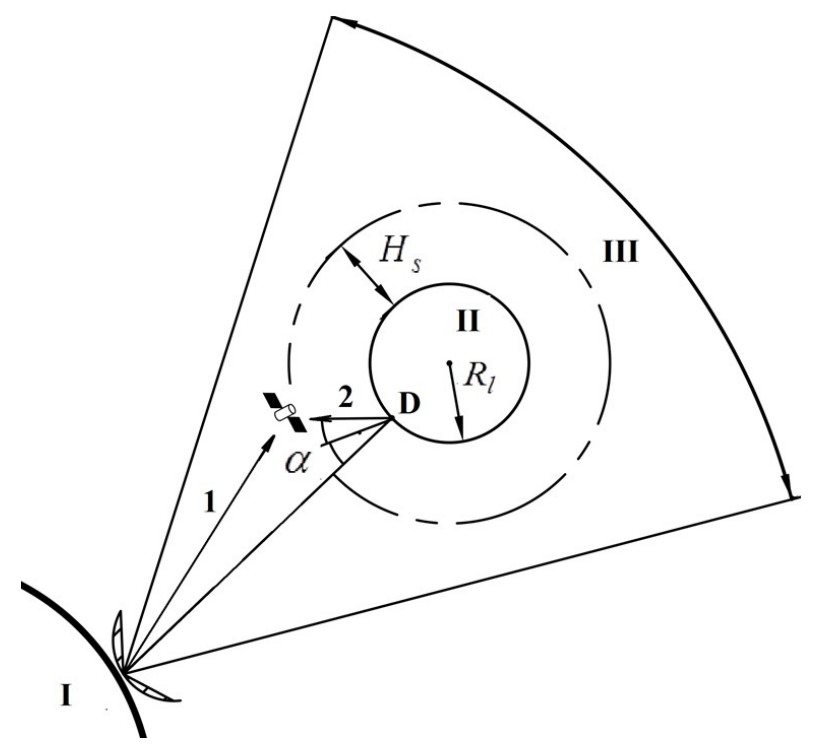

Fig. 1. Sheme of the Moon bistatic radar sounding.
Fig. 1, this angle is marked $\alpha$, and the center of the reflection region is indicated by point D.

The reflection of radio waves from an inhomogeneous surface is usually considered as the sum of two components: diffuse scattering and quasi-specular reflection. When ground-based radar is in operation, the entire surface of the Moon visible from Earth is a region of diffuse scattering. Fig. 2 shows a diagram of the formation of the reflected signal: the signal propagates to the Moon (in the figure it is indicated by the number I) from the Earth (the number is II). The shaded area in the diagram corresponds to the part of the surface involved in the formation of diffuse scattering. The quasi-mirror reflection of radio waves in the remote sensing approximation of radiophysics is modeled by a ray tube whose diameter is associated with two or three Fresnel zones [4]. In Fig. 2, this component is shown in gray. The ray tube limits the most essential

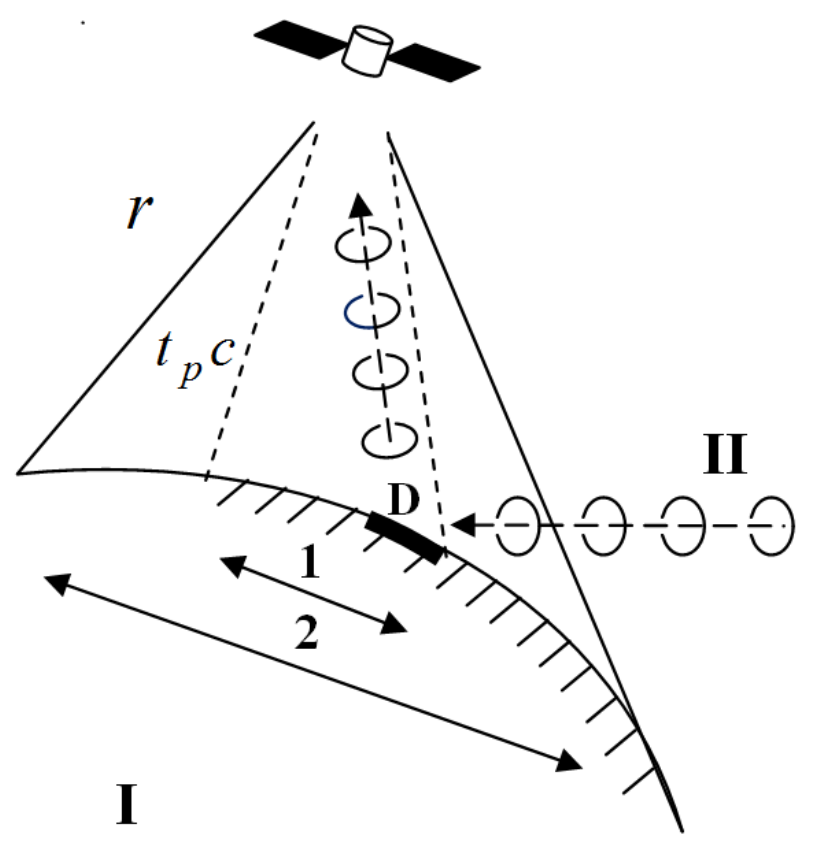

Fig. 2. Radio signal reffection in bistatic radar sounding experiments. 
region for reflection on the surface, in Fig. 2 this surface area is painted black, its center is marked with the letter $D$. Orbital radar can receive a signal reflected from the ground from the territory of diffuse scattering and the area bounded by the ray tube. The time of the work of RLC-L $t_{p}$ will be considered optimal if it received of all quasi-mirror component of the ground-based radar radio signal, but its diffuse component is recorded as little as possible.

For solving the problems of bistatic radar sounding using the orthogonal system of selenographic coordinates (SC) MOON ME. The Moon is approximated by a sphere with a radius $R_{l}=1737.4 \mathrm{~km}$ in this system:

$$
x^{2}+y^{2}+z^{2}=R_{l}^{2} \text {. }
$$

The center of the sphere (1) coincides with the center of mass of the Moon and with the center of the coordinate system MOON ME. Axes $O X$ and $O Y$ lie in the plane of the lunar equator, while the first is directed to the initial meridian, i.e. to the ground. During the experiment, the directions to the Earth and to the groundbased radar coincide. The second axis faces east, toward Mare Crisium. The axis $O Z$ is located along the average position of the axis of rotation of the body and is directed to the North Pole. A spherical system of selenographic coordinates is associated with a three-dimensional orthogonal system, in which the longitude is measured from the zero meridian. The meridians can be numbered in two ways - from $0^{\circ}$ to $360^{\circ}$ east (as when processing the results of NASA lunar missions), or from $0^{\circ}$ to $+180^{\circ}$ east and up to $-180^{\circ}$ west. We will use the second method of counting longitude to preserve the analogy with the earthly mapping technology. Longitude $\varphi$ is defined as the dihedral angle between the plane of the prime meridian and the plane of the meridian passing through the observation point. Selenographic latitude $\theta$ is the length of the arc along the meridian and is measured from the equator (to the north with a plus sign, to the south with a minus sign). The coordinates of the spacecraft in the rectangular and spherical systems are related by the formulas:

$$
\left(\begin{array}{l}
x_{s} \\
y_{s} \\
z_{s}
\end{array}\right)=\left(\begin{array}{l}
\left(R_{l}+H_{s}\right) \cos \theta_{s} \cos \varphi_{s} \\
\left(R_{l}+H_{s}\right) \cos \theta_{s} \sin \varphi_{s} \\
\left(R_{l}+H_{s}\right) \sin \theta_{s}
\end{array}\right) .
$$

For solving posed problem, it suffices to consider the plane passing through the spacecraft, the Moon center and the groundbased radar with coordinates $(L-R ; 0 ; 0)$. Here $L$ is the distance between the Earth center and the Moon one. Denote the plane $\beta$ and find its equation:

$$
\left|\begin{array}{lll}
x & y & z \\
L-R_{e} & 0 & 0 \\
x_{s} & y_{s} & z_{s}
\end{array}\right|=0 .
$$

After the transformation, the equation of the plane $\beta$ will be $y=z \frac{y_{s}}{z_{s}}$ or $y=q \operatorname{ctg} \theta \sin \varphi_{\mathrm{s}}$. It means, that it passes through the axis $O X$ and is tilted to the equatorial plane at an angle $\psi$, whose tangent is $\operatorname{tg} \psi=\operatorname{tg} \theta_{s} / \sin \varphi_{s}$.

In the plane $\beta$ we construct a rectangular SC as follows: the axis $O X$ of this system coincides with the axis $O X$ of the original MOON ME system, and the axis $O Z^{*}$ perpendicular to it is connected to the $O Z$ axis of the MOON ME system as follows:

$$
z=z^{*} \sin \psi=z^{*} \frac{\sin \theta_{s}}{\sqrt{\sin ^{2} \theta_{s}+\cos ^{2} \theta_{s} \sin ^{2} \varphi_{s}}} .
$$

With rectangular SC $X O Z^{*}$ plane $\beta$ is connected polar SC. In this SC the spacecraft position is uniquely determined 
by two parameters: a segment OK, whose length is equal to $R_{l}+H_{s}$, and an angle $\eta$. The geometrical location if the spacecraft is denoted by a point $\mathrm{K}$ on Fig. 3. The angle $\eta$ can be determined from the triangle OEK in the MOON ME with the coordinates of points $O, E\left(R_{l}+H_{s} ; 0 ; 0\right)$, where $E$ is the point of intersection of the zero meridian with the equator, and $\mathrm{K}$. As result we have formula: $\cos \eta=\cos \theta_{\mathrm{s}} \cos \varphi_{\mathrm{s}}$. The maximum value at $\eta$ for altitude in $50 \mathrm{~km}$ is $103^{\circ}$, for $100 \mathrm{~km}-110^{\circ}$.

\section{LOCATION OF THE VISIBILITY ZONE OF THE ORBITAL RADAR AND THE REFLECTING SPOT OF RADIOSIGNAL}

The "radar visibility zone" is a part of the surface bounded by the line of intersection of the sphere (1) and the cone, whose axis coincides with the straight line OK in Fig. 3, and the generator $\mathrm{KF}$ is equal to the distance from the spacecraft to the most distant points of the lunar surface, from which it is still possible take the reflected signal. If the time of reception of the reflected signal is large, then the value is equal to the length of the tangent from the point $\mathrm{K}$ to the circle (Fig. 3); so $r=\sqrt{2 R_{l}^{2}+2 R_{l} H_{s}+H_{s}^{2}}$. The value $r$ depends only on the height of the

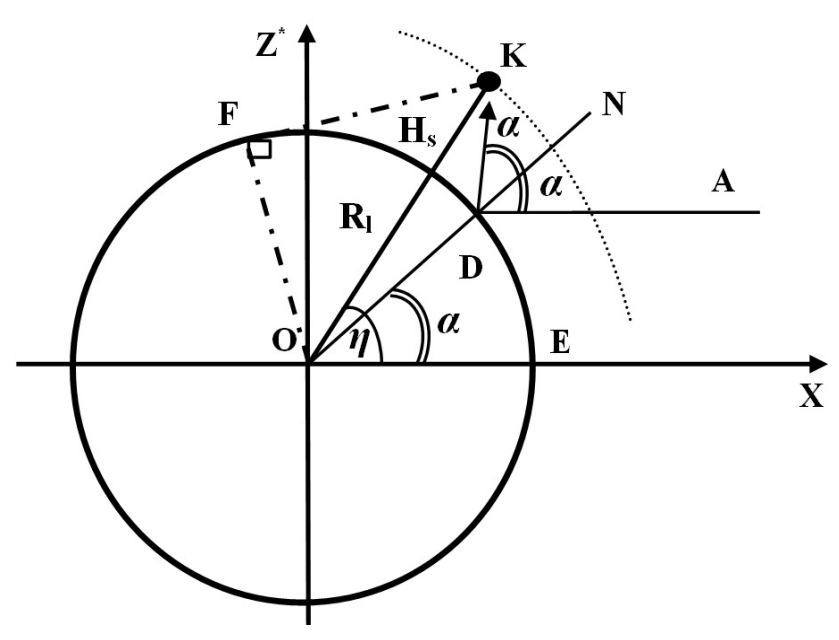

Fig. 3. Rectangular coordinate system of plane $\beta$. apparatus (on Fig. 2 this line corresponds to the solid straight line marked $r$ ). If the registration time is limited as $t_{\mathrm{p}}<r / c$, where $c$ is the speed of light, then the linear dimensions of the visibility zone decrease: the distance to the most distant points of the surface becomes equal $t_{p}$. In Fig.2, the straight line of such a cone, which limits the zone of visibility, is shown by a dashed straight line. The length of the arc along the surface of the Moon in the radar visibility zone is equal to $2 L$, where

$$
L=R_{l} \arccos \left(\frac{\left(R_{l}+H_{s}\right)^{2}+R_{l}^{2}-t_{p}^{2} c^{2}}{2\left(R_{l}+H_{s}\right) R_{l}}\right) .
$$

Next, we discuss how to highlight the area of reflection of the radio signal. The coordinates of point $D$, according to [5], are determined on the basis of the conditions of geometrical optics. It is believed that the radio signal from the IRNR propagates along the straight line $\mathrm{AD}$, parallel to the axis $O X$ to point $D$ on the surface of the Moon, and after reflection from the surface along the line DK (Fig. 3). Point $D$ is such that the angle $\mathrm{ADN}$ is equal to the angle NDK. Due to the parallelism of the $\mathrm{AD}$ and $O X$, the angles of $\mathrm{XOD}, \mathrm{ADN}$ and NDK are equal, we denote angles as $\alpha$. Maximum value of the angle $\alpha$ is $90^{\circ}$.

If the angle $\alpha$ associated with the ballistic coordinates of the spacecraft was known, then in the SC XOZ* the coordinates of point $D$ were determined by the relations:

$x_{\mathrm{D}}=\mathrm{R}_{\mathrm{r}} \cos \alpha$

and

$z_{D}^{*}=R_{l} \sin \alpha$.

In order to find $\alpha$, we consider the triangle KOD on Fig. 3. From the known theorem follows formula:

$\frac{O K}{\sin \angle N D K}=\frac{O D}{\sin \angle O K D}$. 
From $\angle O K D=180^{\circ}-\angle K O D-\angle O D K$, follows $\sin \angle O K D=2 \alpha-\eta$. Thus, the angles $\alpha$ and $\eta$ connected expression:

$$
\left(R_{l}+H_{s}\right) \sin (2 \alpha-\eta)=R_{s} \sin \alpha \text {. }
$$

From equation (4) the value $\alpha$ is found numerically. Equation (4) has several roots, but the physical formulation of the problem is satisfied by the angle located between $0^{\circ}$ and $\eta$.

According to the found value of the angle $\alpha$, the coordinates of point $D$ are determined by expressions (3) in SC XOZ*, and in SC MOON ME according to (2) the coordinates of point $\mathrm{D}$ will look like:

$x_{\mathrm{D}}=\mathrm{R}_{\mathrm{c}} \cos \alpha$

and

$$
y_{D}=\frac{R_{l} \sin \alpha \cos \theta \sin \varphi}{\sqrt{\sin ^{2} \theta+\cos ^{2} \theta \sin ^{2} \varphi}} .
$$

The third coordinate of point $D$ is determined either from equation (1) or from the digital $3 D$ model of the lunar surface, if it is used to analyze the problem.

According to [4], the reflecting spot is an elliptic region; size of the elliptic section is evaluated with the approximate relations:

$$
\Delta y \approx k(s \lambda)^{1 / 2} \cos ^{-1} \eta, \Delta x \approx k(s \lambda)^{1 / 2} \text {. }
$$

Here $\Delta y$ is the linear dimension of the section along the tangent to point $D$ in the plane $\beta$, and $\Delta x$ - one in the perpendicular direction, $\lambda$ is the wavelength for the monochrome signal. Since the IRNR signal is chirp, for the calculations is used $154 \mathrm{MHz}$. The coefficient $k$ specifies the number of Fresnel zones taken into account for estimate the reflection zone. It is usually considered to be 3-4 [4], but in practice and in mathematical modeling this value is taken much more [6]. Formula (6) includes a parameter $s$ that is equal to the distance from the spacecraft to the center of the reflection site. Its value, taking into account (5), is determined by the formula

$$
s=\sqrt{\left(x_{s}-x_{D}\right)^{2}+\left(y_{s}-y_{D}\right)^{2}+\left(z_{s}-z_{D}\right)^{2}} .
$$

\section{TIME OF REGISTRATING OF REFLECTED SIGNAL WITH ORBITAL RADAR}

Next, we consider what conditions the radar operating time should satisfy, so that the region most main for reflection gets into the visibility range of the device.

Point $D$ is the midpoint of the segments $\Delta y$ and $\Delta x$. Due to the fact that $\Delta y<<R_{p}$, the length of the arc of a circle in the plane $\beta$, the ends of which connects this segment, is also equal to $\Delta x$. In this case, the length arch from point under spacecraft to the point farthest from the spacecraft, but still included in the region of the maximum of reflection is equal to the total length of arc corresponding to an angle $\eta-\alpha$ (Fig. 3) and an arc $\Delta y / 2$ corresponding to an angle $\Delta y / 2 R_{\text {p }}$

Thus, the distance between the spacecraft and this point is equal to $R=\sqrt{\left(R_{l}+H_{s}\right)^{2}+\left(R_{l}\right)^{2}-2\left(R_{l}+H_{s}\right) R_{l} \cos \left(\eta-\alpha+k(s \lambda)^{1 / 2} / 2 R_{l} \cos \eta\right)}$. And, therefore, the time of the radar is calculated by the formula: $t_{\mathrm{p}}=c^{-1} \mathrm{R}$.

\section{CONCLUSION}

In the framework of the Russian project "Luna-Resource", the study of the soil of the Moon by the radar complex RLK-L using the bistatic radio sounding method involving the Irkutsk incoherent radar is envisaged. Measurements are planned in the "up link" mode. Based on the Fresnel approximation, the region of greatest reflection is defined as a portion of the surface whose center is found from the condition of equality of angles between the normal to the surface and the directions from the base of the 
normal to the receiver and transmitter. The linear dimensions of the reflection area are associated with two to three Fresnel zones. Measurements are considered optimal if the area of greatest reflection is within the visibility range of the orbital radar. The distance from the spacecraft to the center of the radio reflection region depends on the ballistic coordinates of the spacecraft, and sometimes is 500 kilometers or more, which must be taken into account when planning experiments in determining the time of orbital radar operation and allocating the necessary memory for recording the reflected radio signal. The article presents the derivation of a formula for determining the optimal reception time depending on the location of the spacecraft. A preliminary analysis of the results of numerical simulation of the bistatic radar sounding of the Moon showed that the optimal registration time of the reflected signal depends on the height of the spacecraft and the angle $\eta$, the value of which is related to the longitude and latitude of the spacecraft by the formula $\eta=\arccos \left(\cos \theta_{\mathrm{s}} \cos \varphi_{\mathrm{s}}\right)$. If the angle $\eta$ is greater $60^{\circ}$, only part of the first Fresnel zone involved into the visibility range of the orbital radar. The registration time of the reflected signal in this case is the longest and amounts to $1 \mu$ s with a vehicle height of $100 \mathrm{~km}$.

If the angle $\eta$ belongs to the interval from $8^{\circ}$ to $65^{\circ}$, the time for receiving the signal and, consequently, the amount of memory required to store it is 3-4 times less, and up to 10 Fresnel zones involved into the radar view. The location of the spacecraft in this zone is optimal for carrying out natural measurements.

\section{ACKNOWLEDGMENT}

The work was carried out with partial support of the Basic Research Program No. 12 of the Presidium of the Russian Academy of Sciences "Problems of the Genesis and Evolution of the Universe with the methods of ground-based observations and space research".

\section{REFERENCES}

1. Smirnov VM, Yushkova OV, Marchuk VN, Abramov VV, Kvulinskiy YE, Lyahov YN. Luna-Glob Project: Radio Sounding of the Lunar Soil. Journal of Communications Technology and Electronics, 2013, 58(9):911-918.

2. Yushkova OV, Gavrik AL, Marchuk VN, Yushkov VV, Smirnov VM, Laptev MA, Chernyshev BV, Dutyshev IN, Lebedev VP, Medvedev AV, Petrukovich AA. Bistatic Radar Detection in the LunaResurs Mission. Solar System Research, 2018, 52(4):287-300.

3. Yushkova OV, Yakovlev OI. Analysis of the possibilities to determine lunar soil characteristics with the help of bistatic radar. Journal of Communications Technology and Electronics, 2017, 62(1):22-30.

4. Feinberg EL. Rasprostranenie radiovoln vdol zemnoy poverkhnosti [Propagation of radio waves along the earth's surface]. Moscow, USSR Academy of Sciences Publ., 1961, $496 \mathrm{p}$.

5. Yushkova OV, Rudamenko RA, Yushkov VV Localization of the reflection area in the moon's bistatic sounding. Journal of Radio Electronics, 2018, № 11. Available at http://jre.cplire.ru/jre/jul18/2/text.pdf, DOI 10.30898/1684-1719.2018.7.2 (in Russ.). 
20 OLGA V. YUSHKOVA, VYACHESLAV V. YUSHKOV,

6. SmirnovVM,YushkovaOV,Karachevtseva IP, Nadezhdina IE. The influence of relief on formation of reflected signals of subsurface sounding radar. Solar System Research, 2014, 48(3):176-181. 